\title{
Exponentially Slow Transitions on a Markov Chain: The Frequency of Calcium Sparks
}

\author{
Robert Hinch and S. Jon Chapman \\ Oxford Centre for Industrial and Applied Maths, Mathematical Institute, 24-29 St Giles', Oxford, \\ OX1 3LB, UK.
}

(Received 2 October 2006)

\begin{abstract}
Calcium sparks in cardiac muscle cells occur when a cluster of $\mathrm{Ca}^{2+}$ channels open and release $\mathrm{Ca}^{2+}$ from an internal store. A simplified model of $\mathrm{Ca}^{2+}$ sparks has been developed to describe the dynamics of a cluster of channels, which is of the form of a continuous time Markov chain with nearest neighbour transitions and slowly varying jump functions. The chain displays metastability, whereby the probability distribution of the state of the system evolves exponentially slowly, with one of the metastable states occurring at the boundary. An asymptotic technique for analysing the Master equation (a differentialdifference equation) associated with these Markov chains is developed using the WKB and projection methods. The method is used to re-derive a known result for a standard class of Markov chain displaying metastability, before being applied to the new class of Markov chains associated with the spark model. The mean first passage time between metastable states is calculated and an expression for the frequency of calcium sparks is derived. All asymptotic results are compared with Monte Carlo simulations.
\end{abstract}

\section{Introduction}

Cardiac muscle cells contract when the intracellular $\mathrm{Ca}^{2+}$ concentration rises from a resting level of $0.1 \mu \mathrm{M}$ to $1.0 \mu \mathrm{M}$. The majority of this $\mathrm{Ca}^{2+}$ is released from an internal $\mathrm{Ca}^{2+}$ store, called the sarcoplasmic reticulum (SR), through $\mathrm{Ca}^{2+}$ release channels called ryanodine receptors (RyRs) [1]. The RyRs are situated in clusters of 10-100 on the surface of the SR, with each cell containing approximately 10,000 clusters. A $\mathrm{Ca}^{2+}$ spark occurs when the majority of the RyRs in a single cluster open, generating a $\mathrm{Ca}^{2+}$ current from the SR into the intracellular space [2]. Under physiological conditions, $\mathrm{Ca}^{2+}$ sparks (and transients) are initiated when L-type $\mathrm{Ca}^{2+}$ channels situated on the extracellular membrane next to a cluster of RyRs open [3], raising the local $\mathrm{Ca}^{2+}$ concentration. The $\mathrm{Ca}^{2+}$ binds to the RyRs in the cluster causing them to open and release $\mathrm{Ca}^{2+}$ from the $\mathrm{SR}$ (this process is called $\mathrm{Ca}^{2+}$-induced-Ca ${ }^{2+}$-release). However, under a pathological condition where the $\mathrm{Ca}^{2+}$ concentration in the $\mathrm{SR}$ or intracellular space is raised, it is possible for a $\mathrm{Ca}^{2+}$ spark to occur spontaneously. In this paper, we analyse a simple model of $\mathrm{Ca}^{2+}$ sparks [4] and calculate the frequency of spontaneous sparks as a function of SR and intracellular $\mathrm{Ca}^{2+}$ concentrations. This system is bistable, and a spark can be interpreted as a stochastic transition from the state where all the RyRs are closed to the state where most of the RyRs are open. 
Continuous time Markov chains can be used to model discrete birth-and-death processes [5]. We consider birth-and-death process with only nearest neighbour transitions, but where the rate of transitions is a function of the position on the chain. The model of $\mathrm{Ca}^{2+}$ sparks falls into this category of problems, which also includes models from chemistry [6], statistical physics [7] and queueing theory [8]. We consider a class of Markov chains which demonstrate metastable behaviour in the limit of a large number of states $(N \gg 1)$. Metastability can occur in systems which contain a small parameter $\epsilon=1 / N \ll 1$, and is defined ${ }^{1}$ as dynamical behaviour on time-scales of the order $e^{r / \epsilon}$ where $r>0$ [10]. The Markov chains we consider have two states which are deterministically stable. However, over time-scales of $O\left(e^{r / \epsilon}\right)$ the system can switch between these states. Early work on Markov chains with large numbers of states reduced the Master equation to a local diffusion process [11] by truncating the Kramer-Moyal [6, 12] expansions after the second term to yield a Fokker-Planck equation. These expansions are only locally valid, but give a partial approximate solution for the stationary distribution. An analysis of large deviations on a Markov chain, where effective diffusion and drift coefficients are calculated from all moments in the Kramer-Moyal expansion, has successfully calculated the mean first passage times between stable points away from the boundaries $[13,14,9]$. An asymptotic analysis of the full Master equation, using the WKB method, has successfully been used to calculate the mean first passage times between stable points with several types of boundary behaviour $[15,16]$. However, the form of boundary behaviour in the $\mathrm{Ca}^{2+}$ spark model has not been analysed previously. In addition to analyses of continuous time Markov chains in one dimensions, Fokker-Planck equations with homogeneous diffusion constants in one and more dimensions have been studied [17, 18]. While these models demonstrate exponential ill-conditioning and metastable behaviour, the fact that the diffusion constant is homogeneous means that they cannot describe general Markov chains.

The $\mathrm{Ca}^{2+}$ spark model is outlined in section 2 and is simplified to a Markov chain with a large number of states and metastable dynamics. In sections 3 and 4 , we develop a new technique for analysing the metastable behaviour of Markov chains, and re-derive the an expression for the mean first passage time between metastable states [9]. Our method analyses the metastable behaviour of the Master equation, which is a differentialdifference equation, using the WKB and projection methods. The projection method [10] is a technique which has been developed to analyse metastable behaviour in partial differential equations such as Burgers equation [19] and Cahn-Hilliard equation [20]. In section 5, we apply our technique to the special form of Markov chain originating from the $\mathrm{Ca}^{2+}$ spark model and calculate the mean first passage time from the boundary state. The key difference in the Markov chain originating from the spark model is that the discreteness of the states are essential close to the boundary, so a continuum approximation cannot be used there. This result is then applied to the model of $\mathrm{Ca}^{2+}$ sparks in section 6 and the rate of spontaneous $\mathrm{Ca}^{2+}$ sparks is calculated.

1 Note, in the physics literature this type of behaviour in stochastic systems is often called bistablility [9] and metastability has an alternative definition. However, we use the slow dynamics definition of metastability which is consistent with the literature we use [10]. 


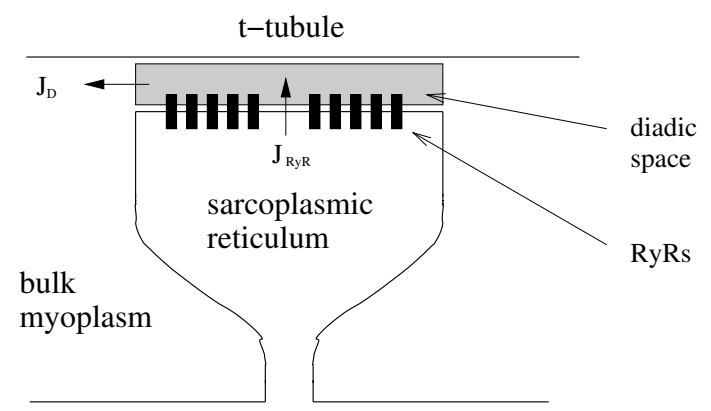

Figure 1. The geometry of a CaRU. Each CaRU contains approximately 50 RyRs.

\section{Calcium Spark Model}

In this section we give a brief introduction to the $\mathrm{Ca}^{2+}$ spark model. Full details of the approximations made and estimates of the model parameters from experimental observations are given in [4]. Cardiac myocytes ${ }^{2}$ contain an internal store of $\mathrm{Ca}^{2+}$ called the sarcoplasmic reticulum (SR), and $\mathrm{Ca}^{2+}$ is released from it through $\mathrm{Ca}^{2+}$ channels called ryanodine receptors (RyRs). The RyRs are situated in clusters of $N \approx 50$ on the surface of the SR, with each cell containing approximately 10,000 separate clusters. The clusters are situated on the SR in positions where the SR meets the t-tubules ${ }^{3}$. There is a small region of the myoplasm ${ }^{4}$, called the diadic space, between the SR and t-tubule membrane which is approximately cylindrically shaped with width $10 \mathrm{~nm}$ and radius $100 \mathrm{~nm}$ [21]. Each cluster of RyRs face into a different diadic space. Additionally, L-type $\mathrm{Ca}^{2+}$ channels situated on the t-tubules face into the diadic spaces, however, they are not involved in the generation of spontaneous $\mathrm{Ca}^{2+}$ sparks due to $\mathrm{Ca}^{2+}$ overload, so are not included in the model. The diadic space and RyRs together make up a $\mathrm{Ca}^{2+}$ release unit (CaRU, see Figure 1). Since the diadic space is a small enclosed volume, its $\mathrm{Ca}^{2+}$ concentration will be higher than the that in the bulk myoplasm when a $\mathrm{Ca}^{2+}$ current passes through the RyRs. The ability of the diadic spaces to support an elevated $\mathrm{Ca}^{2+}$ concentration is the basis of the local control theory of $\mathrm{Ca}^{2+}$-induced-Ca ${ }^{2+}$-release [3]. The $\mathrm{Ca}^{2+}$ concentration in the diadic space is modelled as a compartment, giving

$$
\tau_{\mathrm{ds}} \frac{d c}{d t}=J_{\mathrm{RyR}}+J_{D},
$$

where $c$ is the $\mathrm{Ca}^{2+}$ concentration in the diadic space, $\tau_{\mathrm{ds}}$ is the non-dimensional diffusion time constant of the diadic space, $J_{D}$ is the diffusive current from the diadic space to the bulk myoplasm, $J_{\mathrm{RyR}}$ is the total $\mathrm{Ca}^{2+}$ current through the RyRs, and all quantities have been non-dimensionalised (see [4] for full details). The diffusive current is modelled by a Fickian current

$$
J_{D}=\epsilon_{m}-c,
$$

where $\epsilon_{m}$ is the bulk myoplasmic $\mathrm{Ca}^{2+}$ concentration. The total current through the RyRs is proportional to the number of open RyRs $(n)$ multiplied by the $\mathrm{Ca}^{2+}$ concentration in

2 A cardiac myocyte is another word for a heart muscle cell.

3 T-tubules are invagination of the extracellular membrane into the myocyte.

4 The myoplasm is the intracellular fluid in myocytes. 
the SR

$$
J_{\mathrm{RyR}}=J x
$$

where $x$ is the proportion of channels open $(x=n / N)$ and $J$ is the $\mathrm{Ca}^{2+}$ concentration in the SR. Each RyR contains sites to which $\mathrm{Ca}^{2+}$ ions can bind. When $\mathrm{Ca}^{2+}$ is bound to all these sites the RyR is promoted to a mode where it continuously opens and closes according to a Markov process [22], with a mean open time of $1 \mathrm{~ms}^{5}$. When a RyR opens, an extra $\mathrm{Ca}^{2+}$ current flows from the SR increasing the $\mathrm{Ca}^{2+}$ concentration in the diadic space and increasing the rate at which $\mathrm{Ca}^{2+}$ binds to the other RyRs, thereby creating a positive feedback loop ${ }^{6}$. The RyRs are modelled with a two state Markov model [23] containing a closed state $(C)$ and an open state $(O)^{7}$, with transitions rates

$$
k_{C O}(c)=\frac{1}{k \tau_{\text {open }}} \frac{c^{\alpha}}{c^{\alpha}+1} \quad \text { and } \quad k_{O C}=\frac{1}{\tau_{\text {open }}},
$$

where $k_{C O}(c)$ is the transition rate from the state $C$ to $O, k_{O C}$ is vice versa, $\tau_{\text {open }}$ is the mean open time of a RyR, $\alpha$ is the number of $\mathrm{Ca}^{2+}$ ions needed to bind to each $\mathrm{RyR}$ to open it, and $k$ controls the proportion of time the RyRs are open. The RyRs are assumed to gate independently of each other, but are coupled via the $\mathrm{Ca}^{2+}$ concentration in the diadic space. The mean open time of a RyR is of the order of $1 \mathrm{~ms}$ [22], while the diffusion time of $\mathrm{Ca}^{2+}$ from the diadic space is approximately $3 \mu \mathrm{s}[23]$ so $\tau_{\mathrm{ds}} / \tau_{\text {open }} \approx 0.003$. Therefore the quasi-equilibrium approximation for the $\mathrm{Ca}^{2+}$ concentration in the diadic space can be used. Inserting (2.2) and (2.3) into (2.1) and taking the limit $\tau_{\mathrm{ds}} \rightarrow 0$, yields

$$
c=\epsilon_{m}+J x .
$$

The transition rates of the RyRs (2.4) are now only a function of the proportion of channels open. The model of the CaRU can be reduced to a $N+1$ state Markov chain with nearest neighbour transitions, where each state is labelled by the number of RyRs in the open state $(n)$. Define $F_{n}$ as the jump function (i.e. transition rate) from the $n$ to $n+1$ state and $G_{n}$ as the jump function from the $n$ to $n-1$ state, then

$$
F_{n}=(N-n) k_{C O}\left(\epsilon_{m}+J x\right) \quad \text { and } \quad G_{n}=n k_{O C} .
$$

Finally, defining $f(x)=\tau_{\text {open }} F_{n} / N$ and $g(x)=\tau_{\text {open }} G_{n} / N$ (see [4], equations 19 and 20), yields

$$
f(x)=\frac{\left(\epsilon_{m}+J x\right)^{\alpha}(1-x)}{k\left(1+\left(\epsilon_{m}+J x\right)^{\alpha}\right)} \quad \text { and } \quad g(x)=x .
$$

Physiological estimates of the parameter values are $\epsilon_{m}=0.01, J=4, k=0.2, N=50$ $(\epsilon=0.02), \alpha=4$ and $\tau_{\text {open }}=0.5 \mathrm{~ms}$ [4]. In the rest of the paper we analyse this Markov chain in the limit that the number of RyRs tends to infinity $(N \rightarrow \infty$ and $\epsilon \rightarrow 0)$. The Markov chain (2.7) has the following general properties. It is bistable containing two stable fixed-points ${ }^{8}\left(x_{ \pm}\right)$separated by one unstable fixed-point $\left(x_{0}\right)$. The fixedpoints occur where $f\left(x_{*}\right)=g\left(x_{*}\right)$ and are stable if $f^{\prime}\left(x_{*}\right)<g^{\prime}\left(x_{*}\right)$. The fixed-point $x_{-}$

5 This type of behaviour is called modal gating.

${ }^{6}$ It is this positive feedback loop which leads to the bistability.

7 In reality RyRs also contain inactivated states, however, inactivation does not occur at the beginning of a $\mathrm{Ca}^{2+}$ spark so is not included in this model.

8 These are the fixed-points of the system in the deterministic limit. 
describes the system when a spark is not occurring and the fixed-point $x_{+}$is when a spark is occurring. The frequency of $\mathrm{Ca}^{2+}$ sparks is therefore the reciprocal of the mean first passage time from $x_{-}$to $x_{+}$. When $x \gg \epsilon$, the jump functions (2.7) vary slowly between neighbouring states (i.e. $f(x+\epsilon) / f(x)-1 \sim O(\epsilon)$ ). However, when almost all the receptors are closed $(x \sim \epsilon)$, the jump functions vary by an $O(1)$ amount between neighbouring states (i.e. $f(x+\epsilon) / f(x)-1 \sim O(1)$ ) because $\epsilon_{m} \sim O(\epsilon)$. Additionally, the fixed-point $x_{-}$occurs within the boundary state $x_{-}<\epsilon$. Therefore, although it is possible to consider a continuum limit away from the boundary, it is necessary to consider the discreteness of the chain close to the boundary $x=0$.

Spontaneous $\mathrm{Ca}^{2+}$ sparks are the fundamental release events that make-up $\mathrm{Ca}^{2+}$ waves, which are the origin of certain ectopic heart beats. $\mathrm{Ca}^{2+}$ released from one $\mathrm{CaRU}$ can diffuse to neighbouring CaRUs and triggers further $\mathrm{Ca}^{2+}$ release, thus causing a wave of $\mathrm{Ca}^{2+}$ release to propagate along the myocyte [24]. This mechanism of $\mathrm{Ca}^{2+}$ waves is called the fire-diffuse-fire model [25]. The stochastic properties of $\mathrm{Ca}^{2+}$ sparks are important in determining whether a cell displays $\mathrm{Ca}^{2+}$ waves or global $\mathrm{Ca}^{2+}$ oscillations (i.e. an array enhanced coherence resonance; [26]). The origin of the stochastic nature of $\mathrm{Ca}^{2+}$ sparks lies in the individual receptors, which are either open or closed and switch stochastically between these states. Models of CaRU including L-Type $\mathrm{Ca}^{2+}$ channels have been developed to model $\mathrm{Ca}^{2+}$-induced- $\mathrm{Ca}^{2+}$-release (CICR) in whole myocytes $[27,28]$. These models involve large-scale Monte Carlo simulations of thousands of CaRUs, however, following a similar reduction to that of the spark model, they can be simplified to derive efficient models of the local control of CICR [29, 30].

\section{Asymptotic Analysis of Markov Chains}

Consider an $N+1$ state continuous-time Markov chain with nearest neighbour transitions. Define $f_{n}$ as the jump function (i.e. transition rate) from the $n$ to $n+1$ position on the chain, and $g_{n}$ as the jump function from the $n$ to $n-1$ position on the chain, and let the probability that the system is in the $n^{\text {th }}$ position be $p_{n}(t)$. Then $p_{n}$ satisfies the Master equation

$$
\frac{d p_{n}}{d t}=f_{n-1} p_{n-1}-\left(f_{n}+g_{n}\right) p_{n}+g_{n+1} p_{n+1},
$$

with boundary equations

$$
\begin{aligned}
\frac{d p_{0}}{d t} & =-f_{0} p_{0}+g_{1} p_{1}, \\
\frac{d p_{N}}{d t} & =f_{N-1} p_{N-1}-g_{N} p_{N} .
\end{aligned}
$$

These equations can be in matrix form

$$
\frac{\partial \mathbf{p}}{\partial t}=\mathcal{L} \mathbf{p}
$$

where $\mathbf{p}=\left(p_{0}, \ldots, p_{N}\right)$ and $\mathcal{L}$ is the transition matrix. By summing (3.1) and (3.2) over all $n$, it can be shown that the system conserves probability, i. e.

$$
\frac{d}{d t} \sum_{i=0}^{N} p_{i}(t)=0
$$


Define the relative position on the chain by $x=\epsilon n$, where $\epsilon=1 / N$. Our aim is to analyse the behaviour of this chain in the limit of a large number of states (i. e. as $\epsilon \searrow 0$ ), under the assumption that the jump functions vary slowly with position, so that $f_{n}=f(x)$ and $g_{n}=g(x)$.

The first task is to calculate the steady-state solution of (3.1) and (3.2), which we label $\phi_{n}^{(0)}$. Solving the algebraic equations for $\phi_{n}^{(0)}$ yields

$$
\begin{aligned}
\phi_{n}^{(0)} & =\phi_{0}^{(0)} \prod_{i=0}^{n-1} \frac{f_{i}}{g_{i+1}}, \\
& =\phi_{0}^{(0)} \frac{f_{0}}{g_{1}} \sqrt{\frac{f_{1} g_{1}}{f_{n} g_{n}}} \exp \left(\frac{1}{2} \ln \left(\frac{f_{1}}{g_{1}}\right)+\sum_{i=2}^{n-1} \ln \left(\frac{f_{i}}{g_{i}}\right)+\frac{1}{2} \ln \left(\frac{f_{n}}{g_{n}}\right)\right) .
\end{aligned}
$$

The term in the exponential can be approximated by an integral in the limit $\epsilon \searrow 0$ using the trapezium rule, which is valid providing $\ln \left(f_{n} / g_{n}\right)$ is bounded. This assumption is violated at the boundaries since $g_{0}=0$ and $f_{N}=0$; the solution close to the boundaries will be considered in detail in section 5 . However, the approximation is valid away from the boundaries and we shall first consider cases where the probability that the system is close to the boundaries is negligible. In the limit $\epsilon \searrow 0$, the steady-state distribution is then given by

$$
\phi_{n}^{(0)} \sim \frac{A}{\sqrt{f(x) g(x)}} \exp \left(\frac{1}{\epsilon} \int_{0}^{x} \ln \left(\frac{f(s)}{g(s)}\right) \mathrm{d} s\right),
$$

where $A$ is a constant chosen to satisfy the condition that the total probability is 1 .

We now calculate approximate solutions $\left(\hat{p}^{\epsilon}\right)$ of the transition matrix, which are exponentially accurate, i. e.

$$
\mathcal{L} \hat{p}^{\epsilon} \sim O\left(e^{-r / \epsilon}\right),
$$

where $r>0$, by introducing the WKB-ansatz. These solutions will include the steadystate solution (3.6) and eigen-solutions of the Master equation with exponentially small eigenvalues. At leading order (in $\epsilon$ ), the WKB ansatz solution will fail to uniquely determine the amplitude of the steady-state solution (3.6), and this indeterminacy cannot removed by extending the WKB solution to higher-orders. The problem arises because the transition matrix $\mathcal{L}$ has exponentially small eigenvalues, which correspond to dynamical behaviour over exponentially long time-scales (i. e. metastability). In section 4, we will examine this metastable behaviour using the projection method [10] and derive an expression for the mean first passage time between stable fixed-points away from boundaries. This expression has been derived previously using a different technique [9], which used the WKB method to calculate stationary distributions and analysed the unstable Ornstein-Uhlenbeck process at the unstable fixed-point. In section 5, we extend the analysis to consider the special class of boundary problems which contains the $\mathrm{Ca}^{2+}$ spark model.

In the limit $\epsilon \searrow 0$, we propose the WKB ansatz

$$
\hat{p}_{n}^{\epsilon} \sim R(x ; \epsilon) e^{u(x) / \epsilon} \quad \text { with } \quad R(x ; \epsilon) \sim \sum_{m=0}^{\infty} \epsilon^{m} R_{(m)}(x),
$$

where $x=\epsilon n$. Note, we are approximating the discrete probability density function $\hat{p}_{n}^{\epsilon}$ 
by a continuous function. With this ansatz we find

$$
\hat{p}_{n \pm 1}^{\epsilon}=\hat{p}_{n}^{\epsilon} e^{ \pm u^{\prime}}\left(1 \pm \epsilon \frac{R^{\prime}}{R}+\epsilon \frac{u^{\prime \prime}}{2}+O\left(\epsilon^{2}\right)\right)
$$

where $^{\prime} \equiv \mathrm{d} / \mathrm{d} x$. Inserting (3.9) into the Master equation (3.1) and expanding in powers of $\epsilon$ yields

$$
0=\left(f-\epsilon f^{\prime}\right) e^{-u^{\prime}}\left(1-\epsilon \frac{R^{\prime}}{R}+\epsilon \frac{u^{\prime \prime}}{2}\right)-(f+g)+\left(g+\epsilon g^{\prime}\right) e^{u^{\prime}}\left(1+\epsilon \frac{R^{\prime}}{R}+\epsilon \frac{u^{\prime \prime}}{2}\right)+O\left(\epsilon^{2}\right) .
$$

Equating terms at leading order in $\epsilon$ yields

$$
0=f\left(e^{-u^{\prime}}-1\right)+g\left(e^{u^{\prime}}-1\right) .
$$

Solving this quadratic equation in $e^{u^{\prime}}$ gives

$$
u=\int^{x} \ln \left(\frac{f}{g}\right) \mathrm{d} y \quad \text { or } \quad u=\text { constant. }
$$

The second solution fails when the amplitude function $R_{(0)}(x)$ is calculated so is discarded. Equating terms in (3.10) at $O(\epsilon)$ yields

$$
f e^{-u^{\prime}}\left(-\frac{R_{(0)}^{\prime}}{R_{(0)}}+\frac{u^{\prime \prime}}{2}\right)+g e^{u^{\prime}}\left(\frac{R_{(0)}^{\prime}}{R_{(0)}}+\frac{u^{\prime \prime}}{2}\right)-f^{\prime} e^{-u^{\prime}}+g^{\prime} e^{u^{\prime}}=0,
$$

which becomes

on using (3.12). Hence

$$
\frac{R_{(0)}^{\prime}}{R_{(0)}}+\frac{f^{\prime}}{2 f}+\frac{g^{\prime}}{2 g}=0
$$

and

$$
R_{(0)}=\frac{A}{\sqrt{f g}}
$$

$$
\hat{p}_{n}^{\epsilon} \sim \frac{A}{\sqrt{f g}} \exp \left(\frac{1}{\epsilon} \int_{0}^{x} \ln \left(\frac{f}{g}\right) \mathrm{d} y\right)+O(\epsilon),
$$

where $A$ is a constant to be determined. This equation is identical to the steady-state solution (3.6) calculated in the previous section. However, the right-hand side (r.h.s.) of the leading-order equation from the WKB method (3.11) vanishes when $u^{\prime}=0$, which are turning-points. These turning points occur when $f(x)=g(x)$ (see (3.12)) and correspond to the fixed-points on the chain. Therefore, we must calculate transition layer solutions in the region of the fixed-points, which will give the connection formulae between the WKB solutions separated by the fixed-points.

We consider a chain with 3 fixed-points $0<x_{-}<x_{0}<x_{+}<1$ (note, we are considering the case where $x_{ \pm}$do not occur close to the boundary), with fixed points $x_{ \pm}$stable (so $g^{\prime}\left(x_{ \pm}\right)>f^{\prime}\left(x_{ \pm}\right)$) and the fixed point $x_{0}$ unstable (so $g^{\prime}\left(x_{0}\right)<f^{\prime}\left(x_{0}\right)$ ). The solution in the transition layers is calculated by introducing the local variable

$$
x=x_{*}+\sqrt{\epsilon} z,
$$

and writing $\hat{p}_{n}^{\epsilon}=P(z)$. Substituting (3.17) into the steady-state master equation (3.1) 
and expanding in the limit $\epsilon \searrow 0$ yields

$$
\begin{aligned}
0= & \left(f\left(x_{*}\right)+(\sqrt{\epsilon} z-\epsilon) f^{\prime}\left(x_{*}\right)+\frac{\epsilon z^{2}}{2} f^{\prime \prime}\left(x_{*}\right)\right)\left(P-\sqrt{\epsilon} \frac{d P}{d z}+\frac{\epsilon}{2} \frac{d^{2} P}{d z^{2}}\right) \\
& -\left(f\left(x_{*}\right)+\sqrt{\epsilon} z f^{\prime}\left(x_{*}\right)+\frac{\epsilon z^{2}}{2} f^{\prime \prime}\left(x_{*}\right)+g\left(x_{*}\right)+\sqrt{\epsilon} z g^{\prime}\left(x_{*}\right)+\frac{\epsilon z^{2}}{2} g^{\prime \prime}\left(x_{*}\right)\right) P \\
& +\left(g\left(x_{*}\right)+(\sqrt{\epsilon} z+\epsilon) g^{\prime}\left(x_{*}\right)+\frac{\epsilon z^{2}}{2} g^{\prime \prime}\left(x_{*}\right)\right)\left(P+\sqrt{\epsilon} \frac{d P}{d z}+\frac{\epsilon}{2} \frac{d^{2} P}{d z^{2}}\right)+O\left(\epsilon^{3 / 2}\right), \\
= & \frac{d^{2} P}{d z^{2}}+\gamma_{*}\left(z \frac{d P}{d z}+P\right)+O\left(\epsilon^{3 / 2}\right),
\end{aligned}
$$

where

$$
\gamma_{*} \equiv \frac{g^{\prime}\left(x_{*}\right)-f^{\prime}\left(x_{*}\right)}{f\left(x_{*}\right)} .
$$

Note that $\gamma_{ \pm}>0$ and $\gamma_{0}<0$. Solving gives the solution in each transition layer

$$
P(z)=e^{-\gamma z^{2} / 2}\left(a+b \int^{z} e^{\gamma y^{2} / 2} \mathrm{~d} y\right),
$$

where $a$ and $b$ are constants which are determined by matching with the outer solutions.

\subsubsection{Stable Fixed-Points}

The connection formulae at stable fixed-points $\left(x_{ \pm}\right)$are now calculated. Let $\hat{p}_{L}^{\epsilon}$ be the solution to the left of the fixed-point $\left(x<x_{*}\right)$ with amplitude $A=A_{L}$ (see (3.16)), and $\hat{p}_{R}^{\epsilon}$ be the solution to the right of the fixed-point $\left(x>x_{*}\right)$ with amplitude $A=A_{R}$. To satisfy the constraint that $P(z)>0$ (i. e. negative probabilities are not allowed), we must set $b=0$ in (3.20). The inner and outer solutions can be matched using Van Dyke's matching rule [31] to give the connection formulae

$$
a=\frac{A_{L} e^{u\left(x_{*}\right) / \epsilon}}{\sqrt{f\left(x_{*}\right) g\left(x_{*}\right)}} \quad \text { and } \quad A_{L}=A_{R} .
$$

This agrees with (3.6) where the amplitude does not change across the turning-point.

\subsubsection{Unstable Fixed-Point}

The connection formula at unstable fixed-point $\left(x_{0}\right)$ is now calculated. Let $\hat{p}_{-}^{\epsilon}$ be the solution to the left of the fixed-point $\left(x<x_{0}\right)$ with amplitude $A=A_{-}$(see (3.16)), and $\hat{p}_{+}^{\epsilon}$ be the solution to the right of the fixed-point $\left(x>x_{0}\right)$ with amplitude $A=A_{+}$. The outer solutions (3.16) written in terms of the transition layer variable (3.17) are, in the limit $\epsilon \searrow 0$,

$$
\begin{aligned}
& \hat{p}_{-}^{\epsilon} \sim \frac{A_{-} e^{u\left(x_{0}\right) / \epsilon}}{\sqrt{f\left(x_{0}\right) g\left(x_{0}\right)}} e^{-\gamma_{0} z^{2} / 2}, \\
& \hat{p}_{+}^{\epsilon} \sim \frac{A_{+} e^{u\left(x_{0}\right) / \epsilon}}{\sqrt{f\left(x_{0}\right) g\left(x_{0}\right)}} e^{-\gamma_{0} z^{2} / 2} .
\end{aligned}
$$


These solutions can be matched to the transition layer solution (3.20) using Van Dyke's rule to give

$$
\begin{aligned}
& a=\frac{A_{+} e^{u\left(x_{0}\right) / \epsilon}}{\sqrt{f\left(x_{0}\right) g\left(x_{0}\right)}}, \\
& b=\frac{\left(A_{-}-A_{+}\right) e^{u\left(x_{0}\right) / \epsilon}}{\sqrt{f\left(x_{0}\right) g\left(x_{0}\right)}} \sqrt{\frac{-\gamma_{0}}{2 \pi}} .
\end{aligned}
$$

Note that this matching has not determined the relationship between $A_{-}$and $A_{+}$. Even proceeding in the expansions and matching at higher orders fails to determine the relationship between $A_{-}$and $A_{+}$. This indeterminacy originates from the fact that the transition matrix $\mathcal{L}(3.3)$ has exponentially small eigenvalues, and is only resolved when we consider exponentially small terms (section 4 ).

The final thing to note is that the sum of probability over all states is one, so

$$
\sum_{n=0}^{N} \hat{p}_{n}^{\epsilon}=1
$$

which we have already shown to be conserved by the Master equation (3.4). Define $T_{ \pm}$ by

$$
T_{-}=\frac{1}{A_{-}} \sum_{n=0}^{\left\lfloor x_{0} / \epsilon\right\rfloor} \hat{p}_{n}^{\epsilon} \quad \text { and } \quad T_{+}=\frac{1}{A_{+}} \sum_{n=\left\lceil x_{0} / \epsilon\right\rceil}^{N} \hat{p}_{n}^{\epsilon},
$$

where $\lceil x\rceil$ and $\lfloor x\rfloor$ are $x$ rounded up and down to the nearest integer, and $A_{-}$is the constant in the outer solution (3.16) when $x<x_{0}$ and $A_{+}$is the constant in the outer solution (3.16) when $x>x_{0}$. These sums can be approximated, in the limit $\epsilon \searrow 0$, by replacing the sums with integrals

$$
T_{-} \sim \frac{1}{\epsilon} \int_{0}^{x_{0}} \frac{e^{u(x) / \epsilon}}{\sqrt{f(x) g(x)}} \mathrm{d} x \quad \text { and } \quad T_{-} \sim \frac{1}{\epsilon} \int_{x_{0}}^{1} \frac{e^{u(x) / \epsilon}}{\sqrt{f(x) g(x)}} \mathrm{d} x .
$$

These integrals can then be evaluated, in the limit $\epsilon \searrow 0$, using Laplace's method [31] to yield

$$
T_{ \pm} \sim \frac{e^{u\left(x_{ \pm}\right) / \epsilon}}{f\left(x_{ \pm}\right)} \sqrt{\frac{2 \pi}{\epsilon \gamma_{ \pm}}}
$$

where $\gamma_{ \pm}$are given by (3.19). The probability that the system is in a state $x<x_{0}$ is $P_{-}=A_{-} T_{-}$and a state $x>x_{0}$ is $P_{+}=A_{+} T_{+}$. The equation for conservation of probability (3.24) becomes

$$
A_{-} T_{-}+A_{+} T_{+}=1 \text {. }
$$

Solving for $A_{+}$gives

$$
A_{+}=\frac{1-A_{-} T_{-}}{T_{+}}
$$

which is used to eliminate $A_{+}$in (3.16) and (3.23) leaving one free parameter $A_{-}$. Nonnegativity of $p_{n}$ tells us that $A_{-} \leq 1 / T_{-}$. In the true steady-state eigenfunction $\phi^{(0)}$ (3.6), $A_{-}=A_{+}=A$, from which (3.29) tells us that $A=1 /\left(T_{-}+T_{+}\right)$. 


\section{Metastability and Exponential Asymptotics}

The analysis so far has left one free parameter in the steady-state solution of (3.1). This is because the transition matrix $\mathcal{L}(3.3)$ has eigenvalues which are exponentially small in $\epsilon$ as $\epsilon \searrow 0$. The eigenvalue problem

$$
\mathcal{L} \phi=\lambda \phi
$$

has one zero eigenvalue $\lambda_{0}=0$ with corresponding eigenfunction $\phi^{(0)}$ (the steady-state). Differentiating the WKB solution (3.16) with respect to the undetermined constant $A_{-}$ gives a second function which satisfies $\mathcal{L} \phi=0$ except for an exponentially small error. Following Ward [10], we expect there is an exponentially small eigenvalue $\lambda_{1}$ and corresponding eigenfunction $\phi^{(1)}$, to which this derivative of the WKB solution is a good approximation, so that

$$
\phi^{(1)}(x) \sim \begin{cases}\frac{e^{u / \epsilon}}{\sqrt{f g}} & x<x_{0}, \\ \frac{e^{u\left(x_{0}\right) / \epsilon}}{\sqrt{f\left(x_{0}\right) g\left(x_{0}\right)}} e^{-\gamma_{0} z^{2} / 2}\left(-\frac{T_{-}}{T_{+}}+\sqrt{\frac{-\gamma_{0}}{2 \pi}}\left(1+\frac{T_{-}}{T_{+}}\right) \int_{z}^{\infty} e^{\gamma_{0} y^{2} / 2} \mathrm{~d} y\right) & x \approx x_{0}, \\ -\frac{T_{-}}{T_{+}} \frac{e^{u / \epsilon}}{\sqrt{f g}} & x>x_{0},\end{cases}
$$

where $z=\left(x-x_{0}\right) / \sqrt{\epsilon}$. To evaluate the eigenvalue $\lambda_{1}$, it is easiest to use the eigenfunctions of the adjoint operator corresponding to $\lambda_{0}$ and $\lambda_{1}$. The adjoint problem is

$$
\frac{d \tilde{\phi}_{n}}{d t}=g_{n} \tilde{\phi}_{n-1}-\left(f_{n}+g_{n}\right) \tilde{\phi}_{n}+f_{n} \tilde{\phi}_{n+1},
$$

with boundary equations

$$
\begin{aligned}
\frac{d \tilde{\phi}_{0}}{d t} & =-f_{0} \tilde{\phi}_{0}+f_{0} \tilde{\phi}_{1} \\
\frac{d \tilde{\phi}_{N}}{d t} & =g_{N} \tilde{\phi}_{N-1}-g_{N} \tilde{\phi}_{N} .
\end{aligned}
$$

The adjoint eigenvalue problem can be written in matrix form

$$
\mathcal{L}^{T} \tilde{\phi}=\lambda \tilde{\phi}
$$

The adjoint eigenfunction corresponding to $\lambda_{0}=0$ is simply $\tilde{\phi}^{(0)}=1$. Solving (4.5) away from the turning points for the exponentially small eigenvalue $\lambda_{1}$ similarly gives $\tilde{\phi}^{(1)} \sim$ constant, where the constant can be different in each of the regions separated by the turning points. The solution in the region of the turning points can be calculated by introducing the local inner variable $z(3.17)$ and writing $\tilde{\phi}_{n}^{(1)}=\tilde{\Phi}^{(1)}(z)$. Inserting $(3.17)$ 
into (4.3) and expanding in the limit $\epsilon \searrow 0$ yields

$$
\begin{aligned}
0= & \left(f\left(x_{*}\right)+\sqrt{\epsilon} z f^{\prime}\left(x_{*}\right)+\frac{\epsilon z^{2}}{2} f^{\prime \prime}\left(x_{*}\right)\right)\left(\tilde{\Phi}^{(1)}-\sqrt{\epsilon} \frac{d \tilde{\Phi}^{(1)}}{d z}+\frac{\epsilon}{2} \frac{d^{2} \tilde{\Phi}^{(1)}}{d z^{2}}\right) \\
& -\left(f\left(x_{*}\right)+\sqrt{\epsilon} z f^{\prime}\left(x_{*}\right)+\frac{\epsilon z^{2}}{2} f^{\prime \prime}\left(x_{*}\right)+g\left(x_{*}\right)+\sqrt{\epsilon} z g^{\prime}\left(x_{*}\right)+\frac{\epsilon z^{2}}{2} g^{\prime \prime}\left(x_{*}\right)\right) \tilde{\Phi}^{(1)} \\
& +\left(g\left(x_{*}\right)+\sqrt{\epsilon} z g^{\prime}\left(x_{*}\right)+\frac{\epsilon z^{2}}{2} g^{\prime \prime}\left(x_{*}\right)\right)\left(\tilde{\Phi}^{(1)}+\sqrt{\epsilon} \frac{d \tilde{\Phi}^{(1)}}{d z}+\frac{\epsilon}{2} \frac{d^{2} \tilde{\Phi}^{(1)}}{d z^{2}}\right)+O\left(\epsilon^{3 / 2}\right), \\
= & \frac{d^{2} \tilde{\Phi}^{(1)}}{d z^{2}}-\gamma_{*} z \frac{d \tilde{\Phi}^{(1)}}{d z}+O\left(\epsilon^{3 / 2}\right),
\end{aligned}
$$

where $\gamma_{*}$ is defined by (3.19). Hence

$$
\tilde{\Phi}^{(1)}(z) \sim a+b \int_{-\infty}^{z} e^{s^{2} \gamma / 2} \mathrm{~d} s,
$$

where $a$ and $b$ are determined by matching to the outer solutions. At the stable fixedpoints (where $\gamma_{ \pm}>0$ ), matching to the outer solution yields $b=0$ so $\tilde{\phi}^{(1)}$ is constant across a stable fixed-point. However, at the unstable fixed-point, matching does not uniquely determine $a$ and $b$ (for the same reason that it failed in the primary problem). Thus

$$
\tilde{\phi}^{(1)} \sim \begin{cases}a_{-} & x<x_{0}, \\ a_{-}+\left(a_{+}-a_{-}\right) \sqrt{\frac{-\gamma_{0}}{2 \pi}} \int_{-\infty}^{z} e^{\gamma_{0} y^{2} / 2} \mathrm{~d} y & x \approx x_{0}, \\ a_{+} & x>x_{0} .\end{cases}
$$

The undetermined constants $a_{+}$and $a_{-}$can be found by demanding that $\tilde{\phi}^{(i)}$ satisfies the orthonormality condition

$$
\left(\tilde{\phi}^{(i)}, \phi^{(j)}\right)=\delta_{i j}
$$

which gives

$$
\tilde{\phi}^{(1)} \sim \begin{cases}\frac{T_{+}}{T_{-}\left(T_{-}+T_{+}\right)} & x<x_{0}, \\ \frac{T_{+}}{T_{-}\left(T_{-}+T_{+}\right)}-\frac{1}{T_{-}} \sqrt{\frac{-\gamma_{0}}{2 \pi}} \int_{-\infty}^{z} e^{\gamma_{0} y^{2} / 2} \mathrm{~d} y & x \approx x_{0}, \\ -\frac{1}{T_{+}+T_{-}} & x>x_{0} .\end{cases}
$$

We are now in a position to calculate the exponentially small eigenvalue $\lambda_{1}$. First, we take the inner product of (4.1) with a suitable test function $H$, to give

$$
\left(H, \mathcal{L} \phi^{(1)}\right)=\lambda_{1}\left(H, \phi^{(1)}\right),
$$

where $H$ is any function that is not orthogonal to $\phi^{(1)}$. To find $\lambda_{1}$ we need to evaluate the two inner products in (4.11). Normally it is convenient to use $H=1$ [10], but it cannot be used in this case because it is an eigenfunction of the adjoint problem $\left(i . e . \tilde{\phi}^{(0)}\right.$ and 
therefore orthogonal to $\left.\phi^{(1)}\right)$. Instead we use

$$
H= \begin{cases}1 & x \leq x_{0}, \\ 0 & x>x_{0} .\end{cases}
$$

Note, any test function which is not orthogonal to $\phi^{(1)}$ can be used, (4.12) was chosen for simplicity. Inserting (4.12) into the l.h.s. of (4.11) yields

$$
\sum_{n=0}^{\left\lceil x_{0} / \epsilon\right\rceil} \mathcal{L} \phi^{(1)}(\epsilon n)=-f\left(x_{0}\right) \phi^{(1)}\left(x_{0}\right)+g\left(x_{0}+\epsilon\right) \phi^{(1)}\left(x_{0}+\epsilon\right),
$$

where $\phi^{(1)}(x)$ is evaluated in the transition layer $\left(x \approx x_{0}\right)$ and is given by (4.2). Inserting (4.2) into (4.13) yields

$$
\begin{aligned}
-f\left(x_{0}\right) \phi^{(1)}\left(x_{0}\right)+g\left(x_{0}+\epsilon\right) \phi^{(1)}\left(x_{0}+\epsilon\right) & =f\left(x_{0}\right)\left(\phi^{(1)}(z=\sqrt{\epsilon})-\phi^{(1)}(z=0)\right)+O(\epsilon) \\
& \sim-e^{u\left(x_{0}\right) / \epsilon} \sqrt{\frac{-\gamma_{0} \epsilon}{2 \pi}}\left(1+\frac{T_{-}}{T_{+}}\right) .
\end{aligned}
$$

The r.h.s. of (4.11) can be evaluated using (3.25) and (3.27)

$$
\lambda_{1} \sum_{n=0}^{\left\lceil x_{0} / \epsilon\right\rceil} \phi^{(1)}(\epsilon n)=\lambda_{1} T_{-} \sim \lambda_{1} \frac{e^{u\left(x_{-}\right) / \epsilon}}{f\left(x_{-}\right)} \sqrt{\frac{2 \pi}{\epsilon \gamma_{-}}} .
$$

Equating (4.15) and (4.14) gives the exponentially small eigenvalue

$$
\lambda_{1} \sim-\frac{\epsilon e^{\Delta u_{-} / \epsilon} f\left(x_{-}\right) \sqrt{-\gamma_{0} \gamma_{-}}}{2 \pi}\left(1+\frac{T_{-}}{T_{+}}\right),
$$

where $\Delta u_{-}=u\left(x_{0}\right)-u\left(x_{-}\right)$. This is an important result because it tells us the rate of transitions from the metastable state centred on $x_{-}$. Following Ward ([10], equation 1.17), we seek a solution of the form

$$
p(x, t) \sim \hat{p}^{\epsilon}\left(x ; A_{-}(t)\right) .
$$

(Note that in our case $\hat{p}^{\epsilon}\left(x ; A_{-}(t)\right)$ satisfies the boundary conditions, so additional terms are not needed.) The next step is the projection step ([10], equation 2.17). We take the inner product of (3.3) with the adjoint eigenfunction $\tilde{\phi}^{(1)}$,

$$
\left(\tilde{\phi}^{(1)}, \hat{p}_{t}^{\epsilon}\right)=\left(\tilde{\phi}^{(1)}, \mathcal{L} \hat{p}^{\epsilon}\right)=\left(\mathcal{L}^{T} \tilde{\phi}^{(1)}, \hat{p}^{\epsilon}\right),
$$

where $\mathcal{L}$ is the transition matrix in (3.3). Using (3.16), (3.25) and (4.10) yields a differential equation for $A_{-}$,

$$
\frac{d A_{-}}{d t}=\left|\lambda_{1}\right|\left(\frac{1}{T_{-}+T_{+}}-A_{-}\right),
$$

which describes the metastable evolution of the solution. We first note that as $t \rightarrow \infty$

$$
A_{-}(t) \rightarrow \frac{1}{T_{-}+T_{+}},
$$

which combined with (3.29) tells us that $A_{-}(\infty)=A_{+}(\infty)$, and agrees with the calculation of the steady-state distribution. The second interesting quantity to calculate is the 

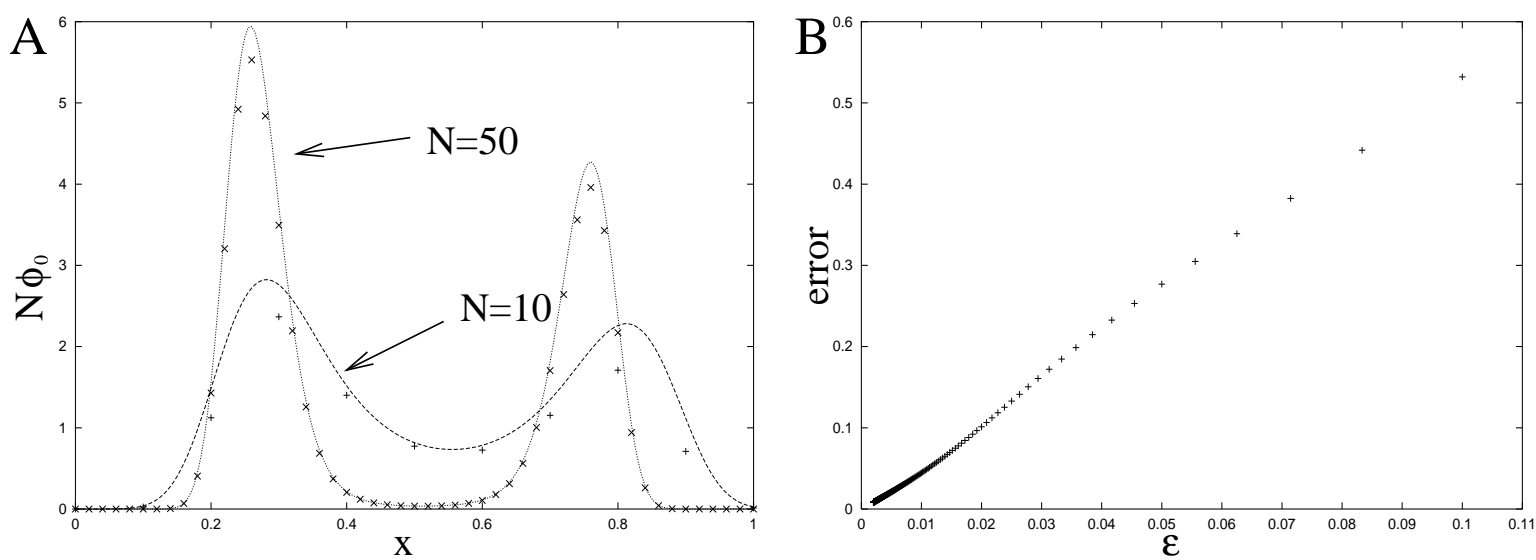

Figure 2. A. Comparison of the asymptotic (lines) and numerical (points) calculation of the stationary distribution of the Markov chain with transition functions (4.23). The stationary distribution was calculated from the mean occupancy states from a Monte Carlo simulation of the chain which included 100,000 (error $\approx 0.3 \%$ ) transitions between the metastable states at $x=0.25$ and $x=0.75$. B. The relative error of the asymptotic result is linear in $\epsilon$.

rate of transfer of probability for the initial value problem where we start with one of $A_{ \pm}=0$. For the case that $A_{+}=0$, the rate at which probability is transfered from the region of $x_{-}$to $x_{+}$is

$$
\frac{d P_{-}}{d t}=T_{-} \frac{d A_{-}}{d t}=-\frac{\epsilon e^{\Delta u_{-} / \epsilon} f\left(x_{-}\right) \sqrt{-\gamma_{0} \gamma_{-}}}{2 \pi},
$$

where we have used (3.29), (4.16) and (4.20). Similarly for the case that $A_{-}=0$, we obtain

$$
\frac{d P_{+}}{d t}=T_{-} \frac{d A_{+}}{d t}=\frac{\epsilon e^{\Delta u_{+} / \epsilon} f\left(x_{+}\right) \sqrt{-\gamma_{0} \gamma_{+}}}{2 \pi} .
$$

These expressions have been derived previously using a different technique [9], and can be compared with Monte Carlo simulations of the transition rates between metastable (fixed-)points on a Markov chain. As a concrete example, we consider a Markov chain with nearest neighbour jump functions

$$
f(x)=(1+x) \exp \left(-100\left\{\left(x-\frac{1}{2}\right)^{3}-\frac{1}{16}\left(x-\frac{1}{2}\right)\right\}\right) \quad \text { and } \quad g(x)=1+x
$$

which has stable fixed points at $x_{-}=1 / 2$ and $x_{+}=3 / 4$, and an unstable fixed-point at $x_{0}=1 / 2$. The argument in the WKB solution (3.12) is simply given by

$$
u(x)=-25\left\{\left(x-\frac{1}{2}\right)^{4}-\frac{1}{8}\left(x-\frac{1}{2}\right)^{2}\right\} .
$$

The stationary distribution $\left(\phi^{(0)}\right)$ can be calculated using the asymptotic formula (3.6) and by directly solving (3.5) numerically. Figure $2 \mathrm{~A}$ shows a comparison of the asymptotic and numerical calculations for $N=10$ and $N=50$; and figure $2 \mathrm{~B}$ shows the relative 

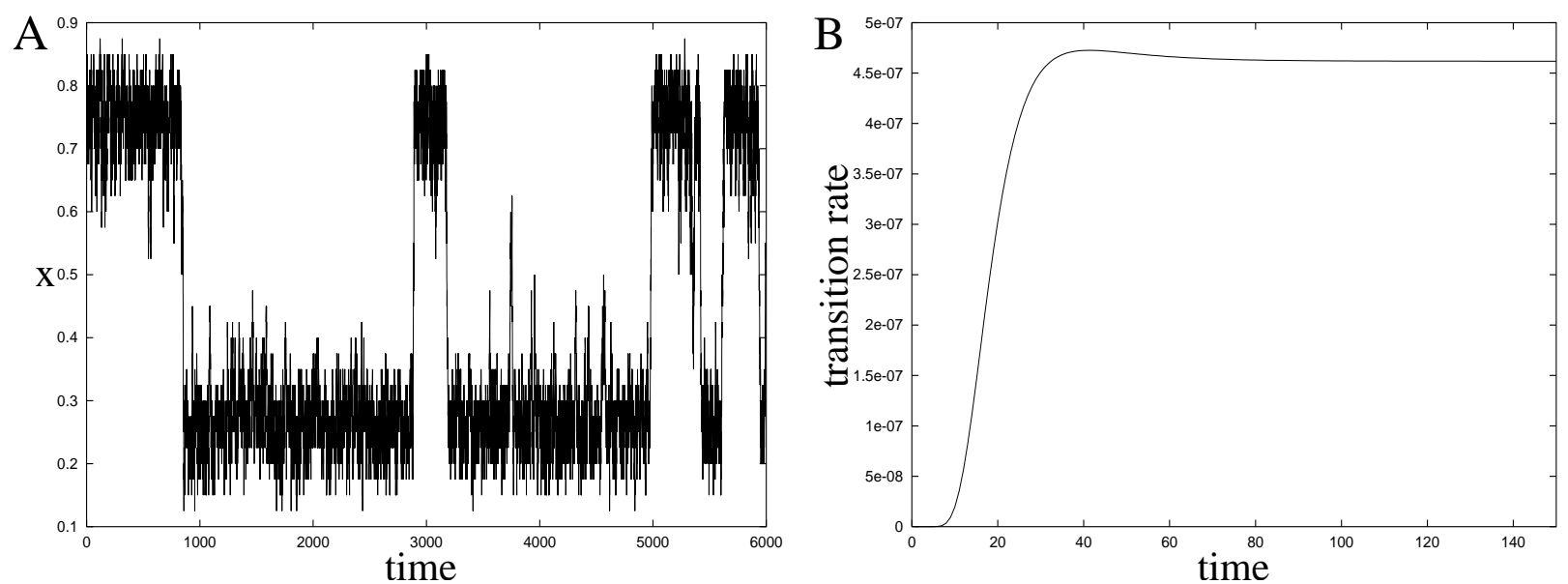

Figure 3. A. Time-series of the position on the Markov chain defined by (4.23) calculated using a Monte Carlo simulation. Note that the transition between the fixed points $\left(x_{ \pm}\right)$is over a much longer time scale than that of the individual transition. B. The transition rate (4.26) from the $x>x_{0}$ region to the $x<x_{0}$ region on the chain calculated by integrating the Master equation. Note that after the initial transients decay the rate of transitions is constant.

error of the asymptotic calculation defined by

$$
\text { error }=\sqrt{\frac{1}{N} \sum_{i=0}^{N}\left(\frac{\phi_{i}^{(0)}(\text { asymptotic })-\phi_{i}^{(0)}(\text { numeric })}{\phi_{i}^{(0)}(\text { asymptotic })}\right)^{2}}
$$

as $\epsilon=1 / N$ is decreased. The relative error in the asymptotic calculation is linear in $\epsilon$. Figure $3 \mathrm{~A}$ shows a typical time series of the position of the system on the Markov chain when $N=30$. Note that the system remains in the region of one of the stable fixed-points $x_{ \pm}$for long times before making a rapid transition to the other stable fixedpoint. The mean first-passage $\left(\tau_{+}\right)$time from $x_{+}$to $x_{-}$was calculated by taking the average of 100,000 (so a $0.3 \%$ error) first-passage times from a Monte Carlo simulation. Computational limitations only allow Monte Carlo simulations to be used when $N<100$ (to calculate the mean first-passage time with the required accuracy when $N=100$ requires $10^{12}$ random numbers to be generated). The mean first-passage time was also calculated by integrating the Master equation (3.1) using a fourth-order Runge-Kutta method with the initial condition $p_{\left\lceil N x_{+}\right\rceil}=1$ and $p_{i}=0$ when $i \neq\left\lceil N x_{+}\right\rceil$. The rate of transitions (i.e. $1 / \tau_{+}$) was calculated using

$$
k_{+}(t)=\frac{1}{\tau_{+}}=\frac{d}{d t} \sum_{i=0}^{\left\lceil N x_{0}\right\rceil} p_{i}(t) .
$$

Figure $3 \mathrm{~B}$ shows $k_{+}$as a function of time. Note that after the initial (algebraic eigenvalues) transients decay, the rate of transitions is approximately constant (in fact it will decay to 0 with the exponentially long time-scale). Figure $4 \mathrm{~A}$ shows the Monte Carlo simulation of the mean first-passage time compared with the asymptotic result (4.22) for different values of $N$. Note that a log-scale is used and that when $N=100$, the mean first-passage time is approximately $10^{6}$. Figure $4 \mathrm{~B}$ shows the relative error of the asymp- 

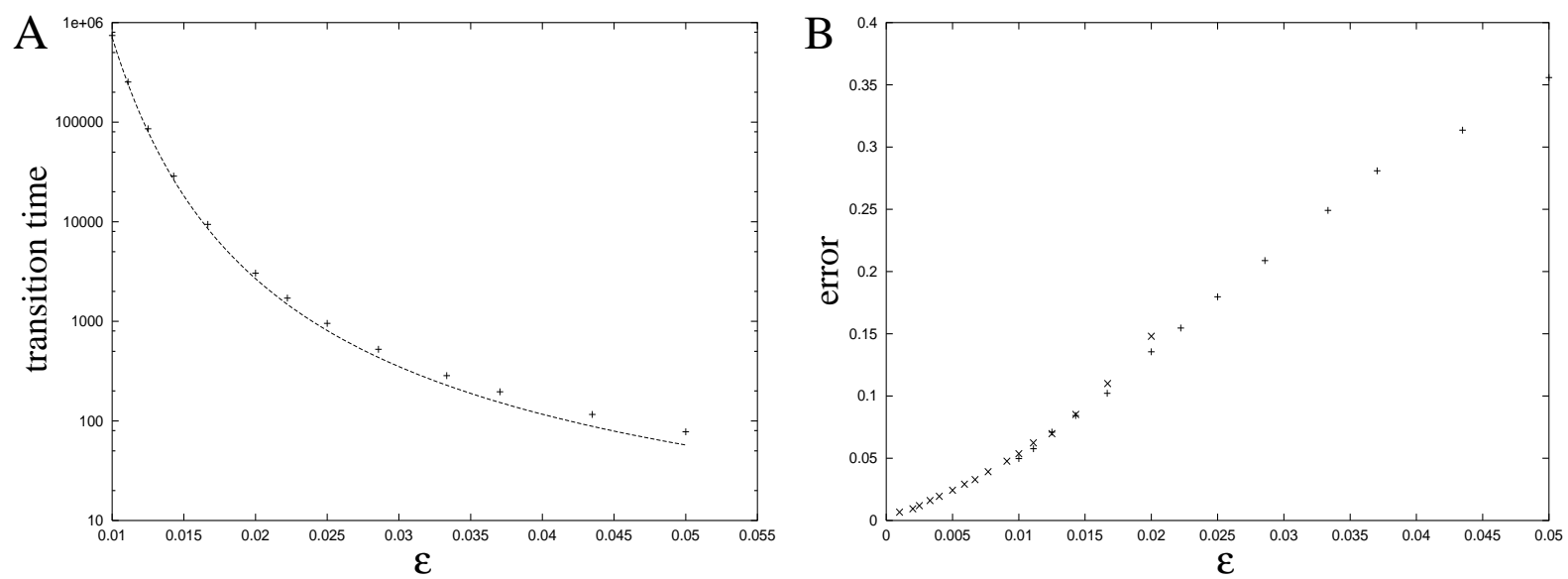

Figure 4. A. A comparison of the Monte Carlo simulation of the mean first-passage time (points) with the analytical approximations (lines; see 4.22). The numerical calculations were performed using a Monte Carlo simulation and averaged over 100,000 transitions to give an accuracy of $0.3 \%$. The $\tau$-axis has a log-scale, so that a wide range of $N$ can be shown. $\mathrm{B}$. The relative error of the asymptotic calculation compared with the numerical calculations (Monte Carlo simulations $(+)$ and integration of the Master equation $(x))$ as a function of the number of states on the Markov chain $(\epsilon=1 / N)$. Note that the error in the asymptotic calculation is linear with $\epsilon$.

totic calculation as compared to the two numerical calculation of the mean first-passage time. Note that the relative error is linear in $\epsilon$.

\section{Fixed-point on the Boundary}

We now discuss a special situation which occurs in the model of calcium sparks (2.7). In the model, one of the stable fixed-points lies on the boundary $x=0$, such that the $\hat{p}_{1}^{\epsilon} / \hat{p}_{0}^{\epsilon} \ll 1$. We consider the case in which the jump functions can be expanded close to the boundary $x=0$ in the form

$$
f(x) \sim(\mu+c x)^{a}+O\left(x^{a+1}\right) \text { and } g(x) \sim x^{b}+O\left(x^{b+1}\right),
$$

where $a>b \geq 1$, and $\rho=\mu / \epsilon c$ is an $O(1)$ constant. Note that the function $f(x)$ contains $\epsilon$ via $\mu$. Using (3.5) tells us that $\hat{p}_{1}^{\epsilon} / \hat{p}_{0}^{\epsilon}=f_{0} / g_{1} \sim \mu^{a} / \epsilon^{b} \ll 1$. The WKB approximation breaks down close to $x=0$ because $\ln (f / g)$ diverges as $x \searrow 0$, and the discrete nature of the Markov chain becomes important. To overcome this problem, we must recalculate $\hat{p}_{n}^{\epsilon}$ in the region of the boundary and match it to the outer WKB solution. The steady-state distribution in the region of the boundary is given by (3.5)

$$
\begin{aligned}
\hat{p}_{m}^{\epsilon} & =\hat{p}_{0}^{\epsilon} \prod_{k=0}^{m-1} \frac{f_{k}}{g_{k+1}}, \\
& \sim \frac{\hat{p}_{0}^{\epsilon} \mu^{a} c^{a m} \epsilon^{(a-b) m}}{\epsilon^{a} c^{a}}\left(\frac{\Gamma(m+\rho)}{\Gamma(1+\rho)}\right)^{a}\left(\frac{1}{\Gamma(1+m)}\right)^{b}\left(\prod_{k=1}^{m-1}(1+O(x))\right) .
\end{aligned}
$$


Define the inner variable $X=\epsilon^{-\beta} x$ and $m=\epsilon^{\beta-1} X$, where $1 / 2<\beta<1$. The product in (5.2) correspond to the higher order terms in the expansion of $f$ and $g$ about $x=0$ :

$$
\prod_{k=1}^{m-1}(1+O(x)) \sim \prod_{k=1}^{\epsilon^{\beta-1} X}\left(1+A X \epsilon^{\beta}\right) \sim 1+O\left(\epsilon^{2 \beta-1}\right),
$$

where $\epsilon^{2 \beta-1} \ll 1$ because $\beta>1 / 2$. Expressing the inner solution in terms of the outer variable, taking the limit $\epsilon \rightarrow 0$ and expanding the gamma functions using Stirling's formula, yields

$$
\begin{aligned}
\hat{p}_{m}^{\epsilon} & \sim \frac{\hat{p}_{0}^{\epsilon} \mu^{a} c^{a x / \epsilon} \epsilon^{(a-b) x / \epsilon}}{\epsilon^{a} c^{a}}\left(\frac{\Gamma(x / \epsilon+\rho)}{\Gamma(1+\rho)}\right)^{a}\left(\frac{1}{\Gamma(1+x / \epsilon)}\right)^{b}, \\
& \sim \frac{\hat{p}_{0}^{\epsilon} \mu^{a} c^{a m} \epsilon^{(a-b) m}(2 \pi)^{(a-b) / 2}}{c \epsilon^{a} \sqrt{m^{a} m^{b}}} \frac{\exp ((a-b)(m \ln m-m)+\rho a \ln m)}{(\Gamma(1+\rho))^{a}},
\end{aligned}
$$

where the second line has been re-expressed in terms of $m$ after the limit $\epsilon \rightarrow 0$ was taken. Expressing the outer WKB solution (3.16) in terms of the inner variable, taking the limit $\epsilon \rightarrow 0$ and performing the integration, yields

$$
\begin{aligned}
\hat{p}_{m}^{\epsilon} & \sim \frac{A_{-}}{\sqrt{\left(f\left(\epsilon^{\beta} X\right) g\left(\epsilon^{\beta} X\right)\right.}} \exp \left(\frac{1}{\epsilon} \int_{0}^{\epsilon^{\beta} X} \ln \left(\frac{f(z)}{g(z)}\right) \mathrm{d} z\right), \\
& \sim \frac{A_{-} \epsilon^{(a-b) m} c^{a m}}{c^{a / 2} \epsilon^{(a+b) / 2} \sqrt{m^{a} m^{b}}} \exp ((a-b)(m \ln m-m)+\rho a \ln m+a \rho(1-\ln \rho)) .
\end{aligned}
$$

Matching (5.4) and (5.5) using Van Dyke's rule, yields

$$
A_{-} \sim \frac{\hat{p}_{0}^{\epsilon} \mu^{a}}{c^{a / 2}}\left(\frac{2 \pi}{\epsilon}\right)^{(a-b) / 2} \frac{\exp (a \rho(\ln \rho-1))}{(\Gamma(1+\rho))^{a}} .
$$

Note $\hat{p}_{0}^{\epsilon}$ is unknown. We now repeat the analysis of section 4 and calculate the rate of transitions from the metastable state $x_{-} \approx 0$. The derivation is identical to that of section 4 except for the evaluation of $T_{-}$:

$$
T_{-}=\frac{1}{A_{-}} \sum_{n=0}^{x_{0} / \epsilon} \hat{p}_{n}^{\epsilon} \sim \frac{\hat{p}_{0}^{\epsilon}}{A_{-}} \sim \frac{c^{a / 2} \epsilon^{(a-b) / 2}}{\mu^{a}(2 \pi)^{(a-b) / 2}} \frac{(\Gamma(1+\rho))^{a}}{\exp (a \rho(\ln \rho-1))} .
$$

The exponentially small eigenvalue $\lambda_{1}$ can be calculated using (4.13) and (4.14):

$$
\lambda_{1} \sim-\frac{\sqrt{-\gamma_{0}} \mu^{a}}{c^{a / 2}}\left(\frac{2 \pi}{\epsilon}\right)^{(a-b-1) / 2} \frac{\exp \left(u\left(x_{0}\right) / \epsilon+a \rho(\ln \rho-1)\right)}{(\Gamma(1+\rho))^{a}}\left(1+\frac{T_{-}}{T_{+}}\right) .
$$

Finally, the transition rate from the metastable state $x_{-}$to $x_{+}$for the initial value problem with $A_{+}(0)=0$ is $(4.21)$

$$
\frac{d P_{-}}{d t} \sim-\frac{\sqrt{-\gamma_{0}} \mu^{a}}{c^{a / 2}}\left(\frac{2 \pi}{\epsilon}\right)^{(a-b-1) / 2} \frac{\exp \left(u\left(x_{0}\right) / \epsilon+a \rho(\ln \rho-1)\right)}{(\Gamma(1+\rho))^{a}} .
$$




\section{The frequency of Calcium sparks}

The analysis of the proceeding section is now applied to the biophysical example of the frequency of $\mathrm{Ca}^{2+}$ sparks (2.7). The jump functions close to $x=0$ are

$$
f \sim\left(\frac{\epsilon_{m}}{k^{1 / \alpha}}+\frac{J x}{k^{1 / \alpha}}\right)^{\alpha} \quad \text { and } \quad g \sim x,
$$

which are of the same form as (5.1) if we set $a=\alpha, b=1, \mu=\epsilon_{m} / k^{1 / \alpha}, c=J / k^{1 / \alpha}$ and $\rho=\epsilon_{m} / \epsilon J$. Using the parameter estimates from experimental observations [4], we find $p_{1} / p_{0} \sim \mu^{a} / \epsilon^{b} \approx 2.5 \times 10^{-6} \ll 1$, so a fixed-point $\left(x_{-}\right)$lies within the state where all the receptors are closed $(n=0)$. When $J>J_{c}$, the chain has two further fixed-points $x_{0}$ and $x_{+}$. Physiologically, the fixed-point $x_{+}$represents the CaRU during a $\mathrm{Ca}^{2+}$ spark. The frequency of sparks is then given by (5.9). To complete this section, we consider the further simplification which arises in the asymptotic limit

$$
\nu=\left(\frac{k}{J^{\alpha}}\right)^{1 /(\alpha-1)} \ll 1 .
$$

Using the parameter values to estimate $\nu$ gives $\nu \approx 0.09$. Note that $\nu \gg \epsilon_{m}$. The position of the unstable fixed-point $\left(x_{0}\right)$ is calculated by setting $f=g$ and is

$$
x_{0} \sim \nu\left(1+\frac{(1+k) \nu}{\alpha-1}-\frac{\epsilon_{m} \alpha}{J(\alpha-1)}\right)+O\left(\nu^{3}, \nu^{2} \epsilon_{m}\right) .
$$

The function $u\left(x_{0}\right)$ is

$$
\begin{aligned}
u\left(x_{0}\right) & =\int_{0}^{x_{0}} \ln \left(\frac{f}{g}\right) \mathrm{d} x, \\
& \sim-(\alpha-1) \nu-\frac{\nu^{2}}{2}+\frac{\alpha \epsilon_{m}}{J}\left(1+\ln \left(\frac{k^{1 /(\alpha-1)}}{J^{1 /(\alpha-1)} \epsilon_{m}}\right)\right)+O\left(\nu^{3}, \epsilon_{m} \nu, \epsilon_{m}^{2} / \nu\right),
\end{aligned}
$$

and $\gamma_{0}$ is

$$
\gamma_{0}=\frac{g^{\prime}\left(x_{0}\right)-f^{\prime}\left(x_{0}\right)}{g\left(x_{0}\right)} \sim-\frac{\alpha-1}{\nu}+O(1) .
$$

The (non-dimensional) spark frequency is then found by inserting (6.2), (6.4) and (6.5) into $(5.9)$

$\frac{d P_{-}}{d t}=-\frac{\sqrt{\alpha-1}}{2 \pi N}\left(\frac{2 \pi N \epsilon_{m}^{2}}{J^{(\alpha-2) /(\alpha-1)} k^{1 /(\alpha-1)}}\right)^{\alpha / 2} \exp \left(-N(\alpha-1)\left(\frac{k}{J^{\alpha}}\right)^{1 /(\alpha-1)}\right) \times A\left(N, k, J, \alpha, \epsilon_{m}\right)$

where

$A\left(N, k, J, \alpha, \epsilon_{m}\right) \sim \exp \left(-\frac{N}{2}\left(\frac{k}{J^{\alpha}}\right)^{2 /(\alpha-1)}+\frac{\alpha \epsilon_{m} N}{J(\alpha-1)} \ln \left(\frac{k}{J^{\alpha}}\right)-\alpha \ln \left(\Gamma\left(\left(1+\frac{N \epsilon_{m}}{J}\right)\right)\right)\right.$.

Note that $A \rightarrow 1$ in the limit $N \nu^{2}, N \epsilon_{m} \rightarrow 0$. This expressions can be checked by comparing it with Monte Carlo simulations of the spark model (figure 5) and the two are in excellent agreement for a wide range of parameters. The physiological significance of these results has been discussed elsewhere [4]. 

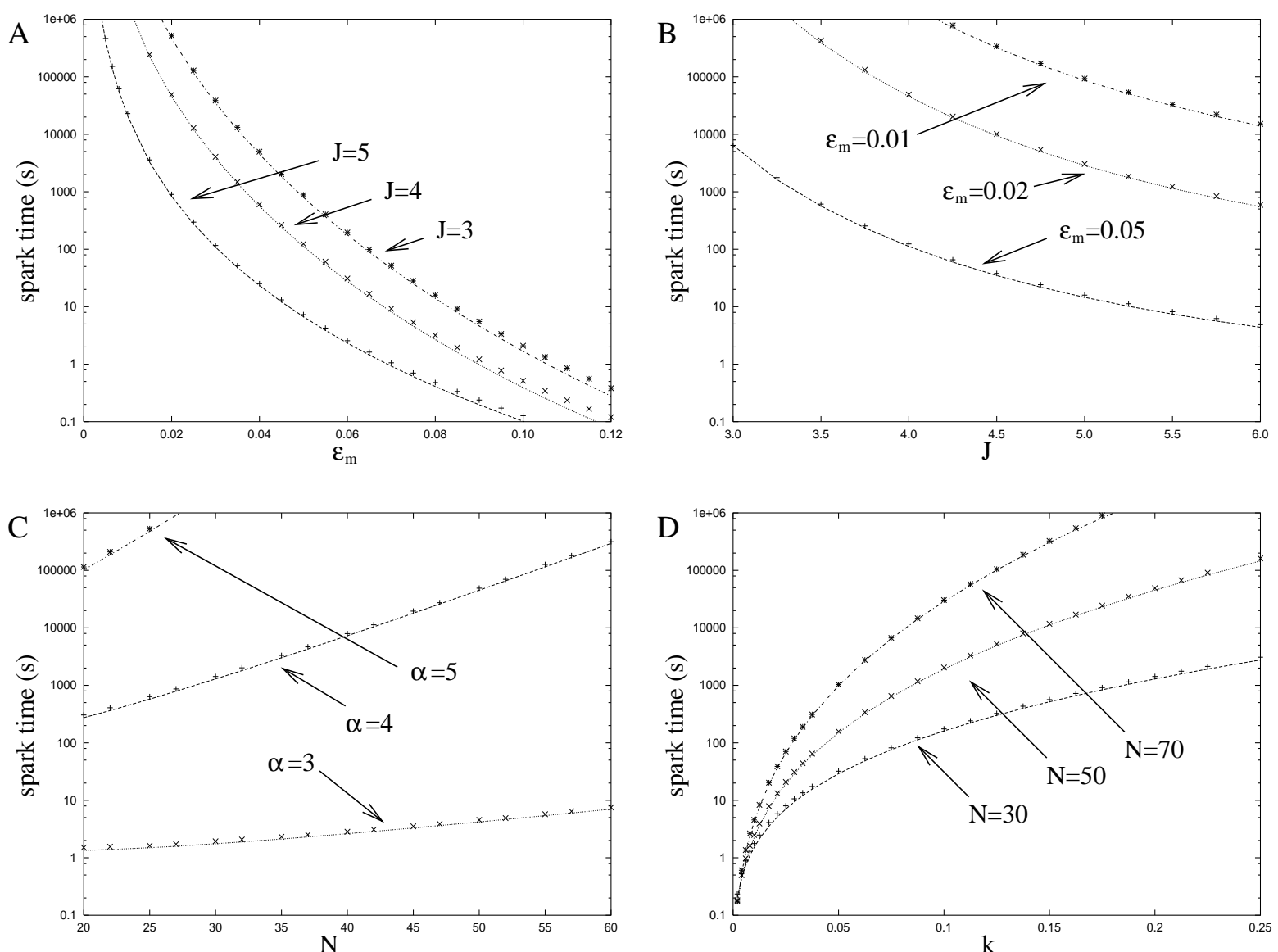

FIgURE 5. The mean spark time, which is the mean first passage time from the fixed-point $x_{-}=0$ to the fixed-point at $x_{+}$. The lines are the asymptotic approximation (6.6) and the points are calculated using a Monte Carlo simulation (averaged over a sample of 10,000). A. The spark time as a function of myoplasmic $\left[\mathrm{Ca}^{2+}\right]\left(\epsilon_{m}\right)$. B. The spark time as a function of SR $\left[\mathrm{Ca}^{2+}\right](J)$. C. The spark time as a function of the number of receptors in the cluster $(N)$. D. The spark time as a function of the proportion of the time the receptor is closed in the H-mode (k). The default parameter used: $N=50, \alpha=4, J=4, k=0.2, \epsilon_{m}=0.02$ and $\tau_{\text {open }}=0.5 \mathrm{~ms}$.

\section{Discussion}

A Ca ${ }^{2+}$ spark is a spontaneous release of $\mathrm{Ca}^{2+}$ from a cluster of RyRs in a cardiac myocyte. A model of $\mathrm{Ca}^{2+}$ sparks was derived and took the form of a Markov chain with a large number of states and slowly varying jump functions. We studied the metastable behaviour of Markov chains with slowly varying jump functions using the Master equation. In particular we considered a chain with two stable fixed points separated by an unstable fixed point. A WKB analysis of the steady state lead to a one parameter family of solutions in which the probability of being in the neighbourhood of either stable fixed point is undetermined. Such solutions are metastable, evolving on an exponentially long decay to the true steady state. The metastability is associated with an exponen- 
tially small eigenvalue of the transition matrix. By using the projection method we were able to calculate this slow evolution. This allowed us to evaluate the exponentially small transition rate from one steady state to the other. The asymptotic calculation was in very good agreement with Monte Carlo simulations. After generalising the result to the case where one of the steady states lies close to the boundary, we applied the method to the model of $\mathrm{Ca}^{2+}$ sparks. The dependence of the frequency of $\mathrm{Ca}^{2+}$ sparks on the myoplasmic and $\mathrm{SR} \mathrm{Ca}^{2+}$ concentrations was calculated.

\section{Acknowledgements}

RH acknowledges the support of The Wellcome Trust, and thanks Prof D. Noble and Dr A. Fowler for useful discussions relating to this work.

\section{References}

[1] D. M. Bers, Excitation contraction coupling and cardiac contractile force, Kluwer Academic Press (Boston), 1993.

[2] H. Cheng, W. J. Lederer, M. B. Cannell, Calcium sparks - elementary events underlying excitation-contraction coupling in heart muscle, Science 262 (1993) 740-744.

[3] M. D. Stern, Theory of excitation-coupling in cardiac muscle., Biophyscial Journal 63 (1992) 469-489.

[4] R. Hinch, A mathematical analysis of the generation and termination of calcium sparks, Biophysical Journal 86(3) (2004) 1293-1307.

[5] G. Grimmett, D. Stirzaker, Probability and Random Processes (3rd edition), Oxford University Press, 2001.

[6] H. A. Kramers, Brownian motion in a field of force and the diffusion model of chemical reactions, Physica VII (1940) 284-304.

[7] N. G. van Kampen, Stochastic processes in Physics and Chemistry, North-Holland, New York, 1981.

[8] G. F. Newell, Application of Queuing Theory, Chapman and Hall (London), 1971.

[9] P. Hanggi, H. Grabert, P. Talkner, H. Thomas, Bistable systems: Master equation versus Fokker-Planck modelling, Phys. Rev. A 29 (1) (1984) 371-378.

[10] M. J. Ward, Analysing multiscale phenomena using singular perturbation methods, Vol. 56 of Proceedings of Symposia in Applied Mathematics, AMS Short Courses, 1998, Ch. Exponential asymptotics and convection-diffusion-reactions models, pp. 151-184.

[11] N. G. van Kampen, A power series expansion of the Master equation, Can. J. Phys. 39 (1961) 551-567.

[12] J. E. Moyal, Stochastic processes and statistical physics, J. Roy. Stat. Soc. (London) B11 (1949) 150-172.

[13] P. Hanggi, Nonlinear fluctuations: The problem of deterministic limit and reconstruction of stochastic dynamics, Phys. Rev. A 25 (1982) 1130-1136.

[14] P. Hanggi, H. Thomas, Stochastic pricesses: Time evolution, symmetries and linear response, Phys. Rep 25 (1982) 1130-1136.

[15] C. Knessl, M. Mangel, B. J. Matkowsky, Z. Schuss, C. Tier, Asymptotic solution of the Kramers-Moyal equation and first passage times for Markov jump processes, Phys. Rev. A 29 (1984) 3359-3369.

[16] C. Knessl, B. J. Matkowsky, Z. Schuss, C. Tier, An asymptotic theory of large deviations for Markov jump processes, SIAM J. Appl. Math. 46 (1985) 1006-1028.

[17] B. J. Matkowsky, Z. Schuss, Eigenvalues of the Fokker-Planck operator and the approach to equilibrium for diffusion fields, SIAM J. Appl. Maths. 40 (2) (1981) 242-254. 
[18] R. S. Maier, D. L. Stein, Effect of focusing of caustics on exit phenomena in systems lacking detailed balance, Phys. Rev. Let. 71 (12) (1993) 1783-1786.

[19] L. G. Reyna, M. J. Ward, On the exponentially slow motion of a viscous shock, Comm. Pure Appl. Math 48 (1995) 79-120.

[20] L. G. Reyna, M. J. Ward, Metastable internal layer dynamics for the viscous Cahn-Hilliard equation, Methods and Appl. of Anal 2 (1995) 285-306.

[21] C. Soeller, M. B. Cannell, Numerical simulations of local $\mathrm{Ca}^{2+}$ movements during l-type $\mathrm{Ca}^{2+}$ channel gating in the cardiac diad., Biophys. J. 73 (1997) 97-111.

[22] A. Zahradnikova, I. Zahradnik, A minimal gating model for the cardiac $\mathrm{Ca}^{2+}$ release channel, Biophysical Journal 71 (1996) 2996-3012.

[23] E. A. Sobie, K. W. Dilly, J. dos Santos Cruz, W. J. Lederer, M. S. Jafri, Termination of $\mathrm{Ca}^{2+}$ sparks: An investigative mathematical mode of $\mathrm{Ca}^{2+}$ induced $\mathrm{Ca}^{2+}$ release in the cardiac diad., Biophysical Journal 83 (2002) 59-78.

[24] H. Cheng, M. R. Lederer, W. J. Lederer, M. B. Cannell, Calcium sparks and $\left[\mathrm{Ca}^{2+}\right]_{\mathrm{i}}$ waves in cardiac myocytes, American Journal of Physiology 270 (1996) C148-C159.

[25] J. E. Keizer, G. D. Smith, S. Ponce Dawson, J. Pearson, Saltatory propagation of $\mathrm{Ca}^{2+}$ waves by $\mathrm{Ca}^{2+}$ sparks, Biophysical Journal 75 (1998) 595-600.

[26] S. Coombes, R. Hinch, Y. Timofeeva, Receptors, sparks and waves in a fire-diffuse-fire framework for calcium release, Prog. Biophys. Mol. Biol. 85 (2004) 197-216.

[27] M. D. Stern, L. S. Song, H. Cheng, J. S. K. Sham, H. T. Yang, K. R. Boheler, E. Rios, Local control models of cardiac excitation-contraction coupling: a possible role for allosteric interactions between ryanodine receptors, J. Gen. Physiol. 113 (1999) 469-489.

[28] J. L. Greenstein, R. L. Winslow, An integrative model of cardiac ventricular myocytes incorporating local control of $\mathrm{Ca}^{2+}$ release, Biophysical Journal 82 (2002) 2918-2945.

[29] R. Hinch, J. L. Greenstein, A. J. Tanskanen, L. Xu, R. L. Winslow, A simplified local control model of $\mathrm{Ca}^{2+}$ induced $\mathrm{Ca}^{2+}$ release in cardiac ventricular myocytes, Biophys. J. 87 (2004) 3723-3736.

[30] R. L. Winslow, R. Hinch, J. L. Greentein, Synapses and Muscles, Springer-Verlag (Berlin), 2005, Ch. Mechanisms and models of cardiac excitation-contraction coupling.

[31] E. J. Hinch, Perturbation Methods, Cambridge University Press, 1991. 